Conclusions: mRNA expression of S100A8, S100A9, S100A12 and TLR4 in PBMCs of TA patients were higher as compared to healthy controls. Despite this, S100A12 secretion was lower in TA as compared to healthy controls upon LPS challenge.

References:

[1] Pryshchep O, Ma-Krupa W, Younge BR, et al (2008) Vessel-specific Toll-like receptor profiles in human medium and large arteries. Circulation 118:12761284.

[2] Deng J, Ma-Krupa W, Gewirtz AT, et al (2009) TLR4 and TLR5 induce distinct types of vasculitis. Circ Res 104:488-495.

Acknowledgements: This study was funded by grants-in-aid from Science and Engineering Research Board (SERB), Department of Science and Technology (DST), Government of India under young investigator scheme to JK (SB/YS/LS14/2014).

Disclosure of Interest: None declared

DOI: 10.1136/annrheumdis-2017-eular.5682

\section{AB0043 TLR4 INHIBITION REDUCES MOVEMENT-INDUCED NOCICEPTION AND ATF-3 EXPRESSION IN EXPERIMENTAL OSTEOARTHRITIS}

J. Ferreira-Gomes ${ }^{1,2,3}$, M.M. Garcia ${ }^{4}$, D. Nascimento ${ }^{1,2,3}$, L. Almeida ${ }^{1,2,3}$, E. Quesada ${ }^{5}$, C. Goicoechea ${ }^{4}$, J. Castro-Lopes ${ }^{1,2,3}$, F. Neto ${ }^{1,2,3} \cdot{ }^{1}$ Departamento de Biomedicina - Unidade de Biologia Experimental, Faculdade de Medicina da Universidade do Porto: ${ }^{2}$ Instituto de Investigação e Inovação em Saúde, Universidade do Porto; ${ }^{3}$ IBMC-Instituto de Biologia Molecular e Celular, Universidade do Porto, Porto, Portugal; ${ }^{4}$ Area de Farmacologia, Facultad de Ciencias de la Salud, Universidad Rey Juan Carlos. Unidad Asociada URJCIQM-CSIC, Alcorcon; ${ }^{5}$ Instituto de Química Médica, Consejo Superior de Investigaciones Científicas. Unidad Asociada URJC- IQM-CSIC, Madrid, Spain

Background: Toll-like receptor 4 (TLR4) is a pattern recognition receptor involved in the initiation of inflammatory responses to control pathogen infections, but is also a "danger-sensing" receptor that recognizes host-derived endogenous molecules called damage-associated molecular patterns (DAMPs). The role of TLR4 in rheumatic diseases is becoming evident, as well as its potential role as a therapeutic option.

Objectives: The aim of this study was to evaluate the effect, both on articular histopathology and pain behaviour, of a TLR4 antagonist (TLR4-A1) on an experimental model of OA. The effect of the TLR4-A1 on the activating transcription factor-3 (ATF-3) signalling pathway was also assessed.

Methods: OA was induced in adult Wistar rats through an intra-articular injection of $2 \mathrm{mg}$ of sodium mono-iodoacetate (MIA) into the left knee. Control animals received a similar injection with saline. TLR4-A1 $(10 \mathrm{mg} / \mathrm{kg})$, synthesized by $\mathrm{Dr}$. Quesada from a compound previously described by the Peri Laboratory (Piazza et al., 2009), was intraperitoneally administered, daily, from days 14 to 28 after $\mathrm{OA}$ induction. Under the same procedure a control group of animals received the vehicle. Movement- and loading- induced nociception was evaluated in all animals, by the Knee-Bend and CatWalk tests, before and after TLR4-A1 or vehicle administration, at several time-points. Animals were sacrificed 28 days after OA induction. L3-L5 Dorsal Root Ganglia (DRG) were used for immunohistochemistry for TLR4 and ATF-3, spinal cords were immunoreacted for TLR4 and knee joints were processed for histopathological evaluation.

Results: Antagonism by TLR4-A1 significantly reduced the nociceptive behavior of $\mathrm{OA}$ animals both in the Knee-Bend and Catwalk tests. The effect was immediately observed 1 day after TLR4-A1 administration, but became more evident 4 days later, maintaining thereafter. No improvement in the cartilage histology was observed. The increased ATF-3 expression observed in DRG of OA animals was significantly reduced by TLR4-A1. On the contrary, TLR4 expression slightly increased after antagonist administration both at DRG and superficial dorsal horn levels.

Conclusions: Chronic treatment with TLR4-A1 showed an antinociceptive effect on $\mathrm{OA}$ animals, not related to articular histopathological improvement, possibly through an ATF-3 dependent mechanism.

References:

[1] Piazza M, Rossini C, Fiorentina SD, Pozzi C, Cornelli F, Bettoni I, Fusi P, Costa $B$ and Peri F. Glycolipids and Benzylammonium Lipids as Novel Antisepsis Agents: Synthesis and Biological Characterization. J Med Chem 2009, 52, 1209-1213.

Acknowledgements: Granted by FEDER funds through COMPETE - Programa Operacional Factores de Competitividade (FCOMP-01-0124-FEDER-021359) and by National Funds through FCT - Fundação para a Ciência e a Tecnologia (PTDC/SAU-NSC/119986/2010); and by Ministerio de Economia y Competitividad (SAF2012-40075-C02-02).

Disclosure of Interest: None declared

DOI: 10.1136/annrheumdis-2017-eular.2878

\section{AB0044 THE INHIBITORY EFFECTS OF TACROLIMUS ON GLIOSTATIN PRODUCTION IN RA SYNOVIOCYTES}

K. Ikuta ${ }^{1}$, Y. Waguri-Nagaya ${ }^{2}$, T. Yamagami ${ }^{3}$, N. Tatematsu ${ }^{3}$, Y. Kawaguchi ${ }^{3}$, Y. Oguri ${ }^{3}$, T. Terazawa ${ }^{1}$, M. Kobayashi ${ }^{3}$, K. Asai ${ }^{4}$, T. Otsuka ${ }^{3} .{ }^{1}$ Orthopaedic
Surgery, Daido Hospital; ${ }^{2}$ Joint Surgery for Rheumatic Diseases: ${ }^{3}$ Orthopaedic Surgery; ${ }^{4}$ Molecular Neurobiology, Nagoya City University Graduate School of Medical Sciences, Nagoya, Japan

Background: Gliostatin (GLS) is known to have angiogenic and arthritogenic activities ${ }^{1}$. We also reported a decrease in serum GLS levels in RA patient responders treated with conventional synthetic disease-modifying anti-rheumatic drugs (csDMARDs) ${ }^{2}$. Tacrolimus (TAC), an orally available calcineurin inhibitor, is a potent immunosuppressant. The efficacy of TAC monotherapy and the combination therapy with TAC and methotrexate in the treatment of RA has been previously described ${ }^{3}$. Although the principal TAC action is thought to be the inhibition of $T$ cell activation, its beneficial effect on RA synoviocytes has not been yet elucidated.

Objectives: To determine the inhibitory effects of TAC on GLS production in RA, we investigated the modulation of serum GLS by TAC therapy and the effect of TAC on the production of GLS in cultured fibroblast-like synoviocytes (FLSs).

Methods: Serum samples were collected from eleven RA patients with active disease at baseline and after 12 weeks of TAC treatment. These RA patients had a history of unsatisfied response with at least one csDMARD or biological DMARD. Serum concentrations of GLS and matrix metalloproteinase (MMP)3 were measured by enzyme immunoassay (EIA). Synovial specimens were obtained from RA patients at the time of total knee arthroplasty. FLSs were cultured and stimulated by TNF alfa with or without TAC. The expression levels of GLS were determined using RT-PCR and EIA. MMP-3 protein in conditioned media was measured by using EIA.

Results: Six patients fulfilled good and moderate responder and the other five patients fulfilled no responder with EULAR response criteria. DAS28, serum GLS, CRP and MMP-3 were significantly down-regulated in TAC responders. In RA FLSs, GLS mRNA and protein were significantly induced after treatment with TNF alfa alone (GLS mRNA 21.9-fold, protein 1.5-fold compared to control). These inductions were suppressed by TAC in a dose-dependent manner. MMP-3 protein was induced by TNF alfa and was similarly suppressed by TAC in a dose-dependent manner (MMP-3 protein 2.9-fold compared to control).

Conclusions: This study is the first to demonstrate that TAC down-regulates GLS mRNA and protein in FLSs treated by TNF alfa. Our data suggests that the beneficial effect of TAC in RA might be due to, at least in part, to anti-angiogenic and anti-arthritogenic activity following the down-regulation of GLS.

References:

[1] Asai K, et al. High concentration of immunoreactive gliostatin/ platelet-derived endothelial cell growth factor in synovial fluid and serum of rheumatoid arthritis. Clin Chim Acta 1993;218:1-4.

[2] Kusabe $\mathrm{T}$, et al. The inhibitory effect of disease-modifying anti-rheumatic drugs and steroids on gliostatin/platelet-derived endothelial cell growth factor production in human fibroblast-like synoviocytes. Rheumatol Int 2005;25:625630.

[3] Kremer JM, et al. Tacrolimus in rheumatoid arthritis patients receiving concomitant methotrexate: a six-month, open-label study. Arthritis Rheum 2003;48:2763-2768.

Acknowledgements: This research was supported by Grand-in-Aid for Scientific Research(C) (26462309) and 16K10913 from the Japan Society for the Promotion of Science.

Disclosure of Interest: K. Ikuta: None declared, Y. Waguri-Nagaya Grant/research support from: Biomet Japan, Inc, Chugai Pharmaceutical Co., Ltd., Astellas Pharma Inc., Merck Sharp \& Dohme Co., AbbVie Japan Co., Ltd., Ono Pharmaceutical Co., Ltd., T. Yamagami: None declared, N. Tatematsu: None declared, Y. Kawaguchi: None declared, Y. Oguri: None declared, T. Terazawa: None declared, M. Kobayashi: None declared, K. Asai: None declared, T. Otsuka: None declared DOI: 10.1136/annrheumdis-2017-eular.2527

\section{AB0045 IL-17 INDUCED GLUCOCORTICOID INSENSITIVITY MIGHT BE DEPENDENT ON THE REDUCED 11BETA-HSD1 ENZYME ACTIVITY}

M. Vitiello ${ }^{1}$, V. Kunnathully ${ }^{1}$, G. Orsolini ${ }^{1}$, O. Viapiana ${ }^{1}$, F. Poli ${ }^{1}$, A. Mattè $^{2}$, L. De Franceschi ${ }^{2}$, L. Idolazzi ${ }^{1}$, D. Gatti ${ }^{1}$, M. Rossini ${ }^{1} .{ }^{1}$ Rheumatology Unit; ${ }^{2}$ Internal Medicine Section, University of Verona, Verona, Italy

Background: It has been demonstrated that IL-17A is able to induce GC insensitivity. Although several studies have reported the role of IL-17 in GC insensitivity the mechanism underlying remains still largely unclear.

Objectives: To understand the effects of interleukin-17 (IL-17) on the enzyme

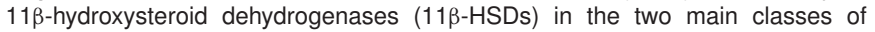
monocytes, CD14 and CD16.

Methods: Peripheral Blood Mononuclear Cells (PBMCs) were isolated from 5 healthy donors and were sorted into CD14 and CD16 subpopulations using cell sorting. Effect of IL-17 on $11 \beta$-HSD1 enzyme activity was measured in terms of conversion of cortisone to cortisol in sorted and unsorted monocytes using Homogeneous Time-Resolved Fluorescence (HTRF). The direct involvement of $11 \beta$-HSD1 in the conversion of cortisone to cortisol was confirmed using carbenoxolone, an inhibitor of $11 \beta$-HSD1.

Results: Monocytes showed a concentration-dependent decrease in the $11 \beta$ HSD1 enzyme activity when incubated with increasing concentrations of IL-17. CD14 and CD16 cells stimulated similarly with IL-17 showed a significant 
difference in the enzyme activity between the untreated and stimulated cells in all treatment groups. However, a dose dependent decrease was observed only in case of CD14 cells. Both unsorted monocytes and monocyte sub-populations showed a significant decrease in the concentration of cortisol measured when co-incubated with carbenoxolone, indicative of the direct involvement of $11 \beta$-HSD1 enzyme in the conversion of cortisone to cortisol.

Conclusions: The results of this study showed that IL-17 induced GC insensitivity might be dependent on the reduced $11 \beta$-HSD1 enzyme activity in inflammatory conditions. We showed that the pro-inflammatory cytokine IL-17 causes a significant decrease in the $11 \beta$-HSD1 enzyme activity.

Disclosure of Interest: None declared

DOI: 10.1136/annrheumdis-2017-eular.2681

\section{AB0046 METABOLISM AND OSTEOARTHRITIS ARE LINKED BY ADIPOKINES}

M.-L. Hülser ${ }^{1}$, C. Schreiyäck ${ }^{1}$, Y. Luo ${ }^{2}$, A. Bozec ${ }^{2}$, G. Schett ${ }^{2}$, E. Neumann ${ }^{1}$, U. Müller-Ladner ${ }^{1} .{ }^{1}$ Internal Medicine and Rheumatology,

Justus-Liebig-University Giessen, Bad Nauheim; ${ }^{2}$ Clinic of Medicine 3 -

Immunology and Rheumatology, University of Erlangen-Nuremberg, Erlangen, Germany

Background: Obesity and hyperinsulinemia are of increasing importance in the Western society. Both obesity and insulin resistance lead to changes in expression of adipokines such as adiponectin, visfatin or leptin, which appear to be immunomodulatory factors also in rheumatic diseases.

Objectives: Since osteoarthritis (OA) is often accompanied by hyperinsulinemia and obesity, we combined both mouse models (destabilization of the medial meniscus (DMM) and high-fat diet (HFD)). Here, we evaluated and correlated the systemic and local effects of both models at different states of OA development with special focus on the local/systemic expression of the adipokines adiponectin, visfatin and leptin over time.

Methods: HFD (mainly consisting of saturated fatty acids) to induce obesity and hyperinsulinemia, and ND (normal diet) as control were fed to C57BI/6 mice for 3 months followed by surgical OA induction (time point 0 ). Tissues and sera were collected at different time points after DMM-mediated OA induction (4, 6, 8 weeks). Adipocytokine (leptin, visfatin, adiponectin and IL-6) serum levels were measured by ELISA. Histological stainings of the joints $(H / E$, safranin $O$, pappenheim and Masson-Goldner's trichrome) were evaluated and arthritis progress was scored. Immunohistochemical stainings of the joints were performed to evaluate the local distribution of adipokines, which were correlated to systemic adipocytokine levels and the respective arthritis score.

Results: Low systemic IL-6 levels confirmed that no acute inflammation due to surgery or infection was present in all animals. OA induction was visible at all time points, which was aggravated in HFD compared to ND mice (OA score: 4 weeks ND 0.87 vs. HFD $0.93,6$ weeks ND 1.44 vs. HFD $3.69,8$ weeks ND 1.78 vs. HFD 2.18). Systemic levels of leptin were significantly induced by HFD confirming the induction of insulin resistance, but DMM decreased leptin levels at all time points (significantly for 3 out of 6 groups, e.g. 4 weeks: HFD healthy vs. HFD DMM 18.4 $\mathrm{ng} / \mathrm{ml}$ vs. $3.7 \mathrm{ng} / \mathrm{ml}$ ). Interestingly, the systemic increase of adiponectin by DMM was time dependent (only 8 weeks after surgery: HFD healthy vs. HFD DMM 5176 $\mathrm{ng} / \mathrm{ml}$ vs. $6149 \mathrm{ng} / \mathrm{ml}$ ) but independent of diet. However, HFD in combination with DMM did not show significant effects on systemic levels of adiponectin, visfatin or IL-6.

Conclusions: HFD deteriorates OA in the DMM model. Systemic leptin levels were elevated by HFD/insulin resistance but reduced by DMM, which could not be observed for the mainly proinflammatory adipokine visfatin. Of note, systemic inflammation as shown by systemic IL- 6 levels was low in all animals. The stage of $\mathrm{OA}$ development influences adiponectin levels, which were only increased systemically 8 weeks after surgery. In summary, systemic levels of adipokines are altered by DMM and HFD as well as the combination of both models and the analyzed adipokines show differing reactions to these factors.

Disclosure of Interest: None declared

DOI: 10.1136/annrheumdis-2017-eular.4913

\section{AB0047 TYPE I INTERFERON IS HIGHLY EXPRESSED IN RA SYNOVIAL FLUID AND JOINT CARTILAGE CORRELATED WITH SERUM RHEUMATOID FACTOR; A PRELIMINARY EXPERIMENTAL STUDY}

M. Nakayama ${ }^{1}$, H. Tobimatsu ${ }^{1}$, H. Imamura ${ }^{1}$, Y. Sakuma ${ }^{1}$, K. Yano ${ }^{1}$, Y. Niki ${ }^{2}$, K. Ikari ${ }^{1} .{ }^{1}$ Department of Orthopedic Surgery, Institute of Rheumatology, Tokyo Women's Medical University; ${ }^{2}$ Department of Orthopedic Surgery, Keio University, Tokyo, Japan

Background: It was reported that type I interferon (IFN) is involved in the pathogenesis of rheumatoid arthritis (RA) [1-3], while the relevance of the IFN signature to RA disease activity and progression remains unclear. There were few reports about the expression of IFN in synovial fluid and joint cartilage.

Objectives: The aim of this study is to investigate the role of IFN in the pathogenesis of RA by means of the analysis of joint tissues of RA patients comparing with those of osteoarthritis $(\mathrm{OA})$ patients.

Methods: Synovial fluid, synovia and cartilage were collected from RA and
OA patients ( $\mathrm{n}=10$ for each) during total knee arthroplasty in our hospital and blood samples were collected just before surgery. As preoperative therapy for RA, Methotrexate (MTX) was administered to 9 patients (dose ranged (4-12mg), DMARDS without MTX to 2, biological DMARDS to 2, Prednisolone (PSL) to 6 (dose $1-5 \mathrm{mg}$ ). Quantities of IFN alpha or beta of blood and joint fluid were measured with ELISA (PBL Assay Science, USA), and expression of IFN alpha, beta and TNF alpha of synovia and joint cartilage were measured with real time PCR. In RA patients. Serum biomarkers such as C-reactive protein (CRP), erythrocyte sedimentation rate (ESR), Rheumatoid Factor (RF), hemoglobin and platelet were measured and investigated correlation with the quantities of IFN of blood or joint tissues. Medication for RA were investigated as well.

Results: In blood and synovial fluid of RA patients, IFN alpha and beta were highly detected, while they were not detected in those of OA patients. The expression of IFN alpha and beta of RA cartilage were much higher than those of OA whereas they were not expressed in synovium of both RA and OA. Expression of IFN was not correlated with that of TNF alpha in RA patients. Statistical analysis revealed that RF was related with blood and joint IFN and other markers were not. Medications for RA were not correlated with IFN expression.

Conclusions: IFN was highly expressed in RA synovial fluid, joint cartilage and blood, not in OA. IFN immunotherapy has been reported to induce RA [4-5], therefore abundant IFN might induce RA and inhibit cure of RA. Our results showed that RF was related with blood and joint IFN. It can be speculated that $\mathrm{RF}$ might be an index of IFN regulation in RA patient, however, more samples must be investigated to prove this speculation.

References:

[1] de Padiila CML and Niewold TB. The Type I Interferons: Basic Concepts and Clinical Relevance in Immune-mediated Inflammatory Diseases. Gene. 2016; $15,576,14-21$

[2] Carrio JR, de Paz B, et al. IFN alpha Serum Levels Are Associated with Endothelial Progenitor Cells Imbalance and Disease Features in Rheumatoid Arthritis Patients. Plos One, 2014;9, e86069.

[3] van Holten J, Smeets TJM, et al. Expression of interferon b in synovial tissue from patients with rheumatoid arthritis: comparison with patients with osteoarthritis and reactive arthritis. Ann Rheum Dis. 2005; 64: 1780-2.

[4] Passos SE, Evangelista SPT, et al. Rheumatoid arthritis induced by alphainterferon therapy. Clin Rheumatol. 2001;20(4):297-9.

[5] Cacopardo B, Benanti F, et al. Rheumatoid arthritis following PEG-interferonalfa-2a plus ribavirin treatment for chronic hepatitis $\mathrm{C}$ : a case report and review of the literature. BMC research notes. 2013; 6:437.

Disclosure of Interest: None declared

DOI: 10.1136/annrheumdis-2017-eular.4978

\section{AB0048 ANTIPHOSPHOLIPID ANTIBODIES, INTERLEUKIN-6 AND TUMOR NECROSIS FACTOR- $\alpha$ IN ATHEROSCLEROTIC PROCESS IN PATIENTS WITH RHEUMATOID ARTHRITIS AND SYSTEMIC LUPUS ERYTHEMATOSUS}

N.A. Bashlakova ${ }^{1}$, T.D. Tyabut ${ }^{2}$, A.E. Buglova ${ }^{2} .{ }^{1}$ Ultrasound Diagnostics Department; ${ }^{2}$ Cardiology and Rheumatology Department, Belarusian Medical Academy of Postgraduate Education, Minsk, Belarus

Background: Systemic inflammation has been postulated to be an independent cardiovascular risk factor, particularly in patients with autoimmune rheumatic disorders (ARD), such as rheumatoid arthritis (RA) and systemic lupus erythematosus (SLE), and is associated with accelerated atherosclerosis. There is some evidence to suggest that antiphospholipid antibodies (aPL) may also play a role in the development of atherosclerosis. However, it is few data about the relationship between these autoantibodies and inflammatory mediators in the development of atherosclerosis

Objectives: To clarify the involvement of inflammatory mediators and aPL in the atherosclerotic process in patients with ARD.

Methods: The study included 87 female patients with ARD (RA ( $n=47)$, mean age $45,0(33,0 ; 51,0)$ years old, disease duration $9,0(3,0 ; 14,0)$ years, disease activity (DAS28 $=5,37(4,69 ; 5,86)$ points); SLE $(n=40)$, mean age $33,5(27,5 ; 44,5)$ years old, disease duration $8,0(5,0 ; 14,5)$ years, disease activity SLEDAI-2K 7,0 (4,0; $11,5)$ points). Sixty healthy women (mean age $40,5(36,0 ; 47,0)$ years old) formed the control group.

The levels of high sensitive C-reactive protein (hs-CRP), interleukin-6 (IL-6), tumor necrosis factor - $\alpha$ (TNF- $\alpha)$, LA, IgG/lgM antibodies to cardiolipin (aCL), 32-glucoprotein-1(aß2-GP1), annexin $\mathrm{V}(\mathrm{aAnV})$ and prothrombin (aPT)) were determined with ELISA. Intima-media thickness (IMT) of the carotid artery wall and the presence of atherosclerotic plaques were revealed ultrasonographically according to the described ESH/ESC Guidelines.

Results: The levels of hs-CRP, IL-6, TNF- $\alpha$ were significantly higher in ARD patients than in the control group, which indicates the disease activity. Furthermore, the patients with SLE had a significant correlation between IL- 6 and SLEDAI-2K $(r=0,471, p=0,002)$, TNF $-\alpha$ and SLEDAI-2K $(r=0,499, p=0,001)$, whereas the patients with RA had only significant correlation between hs-CRP and DAS28 $(r=0,355, p=0,031)$.

The concentration of $\lg \mathrm{aCL}$, IgG and $\lg \mathrm{a}$ a 2-GP1, IgM aAnV, IgG aPT, LA were higher in patients with SLE than in the control group, and the levels of IgG and $\lg M a C L$, IgM aß2-GP1, IgG and IgM aAnV, LA were higher in patients with RA v.s. the control group. 